\title{
Fault Zone Complexity and Earthquake Ruptures
}

\author{
by Ryosuke Ando and Teruo Yamashita
}

doi:10.2204/iodp.sd.s01.18.2007

Earthquake ruptures are propagated inside fault zones that consist of structures spanning a wide spectrum of lengths. A natural fault is known to consist of hierarchy structure ranging from the scale of fine grains forming a gauge or ultracataclasite zone along the principal slip plane (Fig. 1, top) up to that of fault system consisting of discrete fault segments (Fig. 1, bottom) and/or branched fault segments (Fig. 1, middle and bottom). Although the physics of earthquakes has been well studied on microscopic and macroscopic scales (Fig. 1, top and bottom, respectively) based on laboratory experiments and seismic inversion analysis, respectively, the effects on a mesoscopic scale (Fig. 1, middle) are poorly understood. Since these structures interact with earthquake ruptures, one of the seismologically fundamental problems is to understand how they are formed and evolved associated with the earthquakes that are the most intensive activities on fault zones.

In this study we focus on the investigation of the formation of branches in mesoscopic and macroscopic scales, and their effects on dynamic ruptures. In our model, branch fault segments are assumed to be nucleated at some discrete points on the main fault, which implies that the main fault is weak locally at these points. Each branch is assumed to be nucleated at each prescribed point on the main fault once the main fault tip passes, and the following fracture criterion is satisfied there. We also assume branches are extending in the direction of the maximum shear traction at each branch. Our studies rely on a simple slip-weakening law as the fracture criterion and the friction law:

$$
\tau(S)=\frac{\tau_{p}-\tau_{r}}{D_{c}}\left(D_{c}-S\right) H\left(D_{c}-S\right)+\tau_{r}
$$

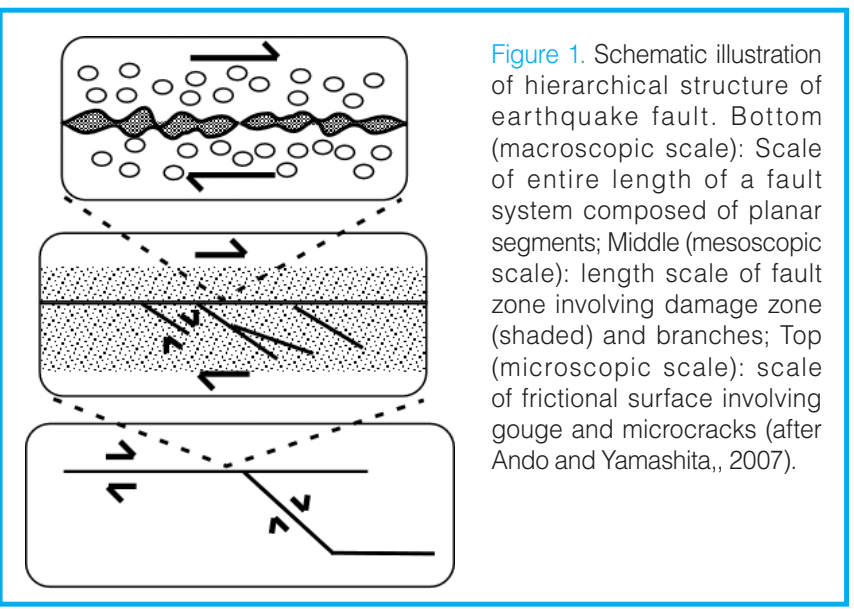

where $\tau_{\mathrm{p}}, \tau_{\mathrm{r}}, S, D_{\mathrm{c}}$, and $H$ (s) denote the peak shear strength, residual shear stress, slip, critical slip displacement, and a unit step function, respectively. The boundary integral equation method (BIEM) is employed to numerically solve the elastodynamic response of the medium. This method has advantages (in the treatment of non-planar fault geometry) and problems (where rupture paths are not specified but dynamically determined as the ruptures are extended).

Figure 2 shows snapshots of a typical example of fault growth. First, the main fault starts its extension and begins to nucleate branches. However, the nucleated branches do not begin their growths when the extension of the main fault is not long enough because of insufficient enhancement of stress (Fig. 2A). As the size of the main fault increases, the shear stress is magnified near its extending tip. If such stress enhancement becomes sufficiently large, branches can begin their growths while their extensions are arrested after attaining certain lengths (Fig. $2 \mathrm{~B}$ and $\mathrm{C}$ ). The spatial distribution of these arrested branches forms a roughly triangular zone on one side of the main fault. The lengths of these branches increase with main fault size. As the main fault extends further and its size exceeds a certain critical length $L_{m}$, a limited number of branches continue to grow together

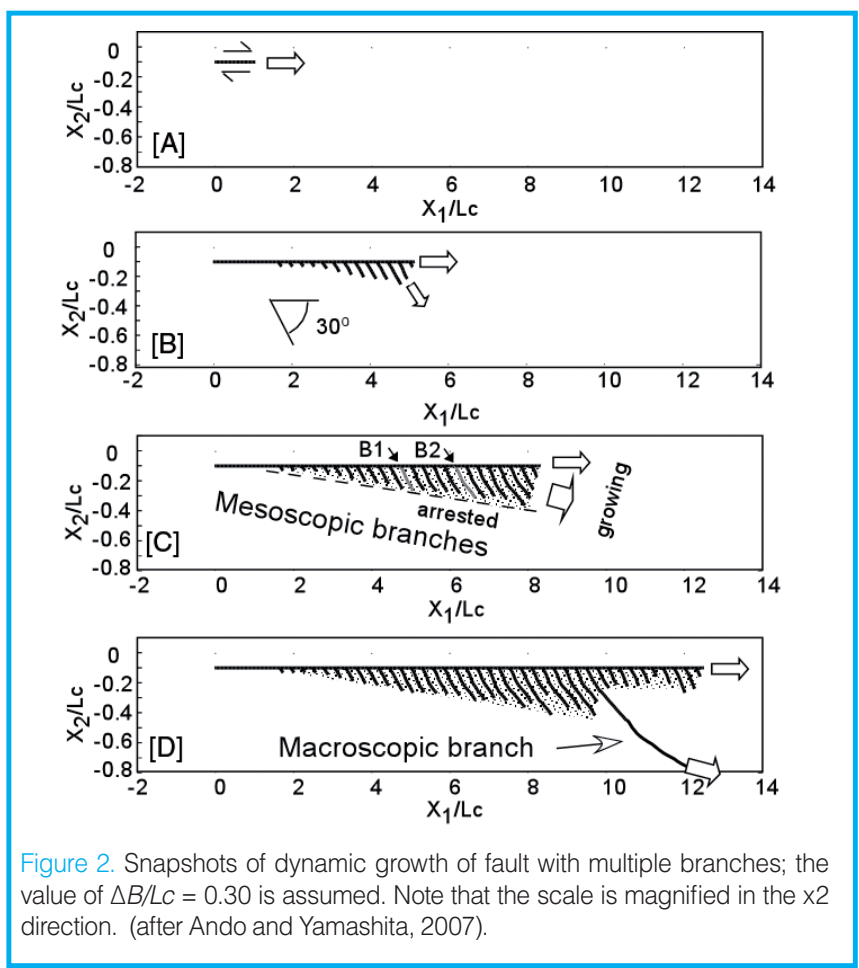



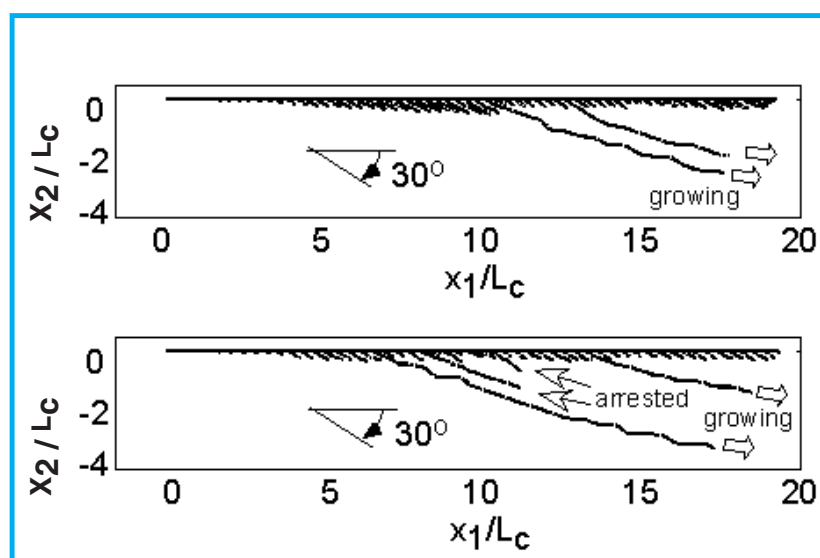

Figure 3. Examples of longer computation. We assume $\Delta B / L C=0.31$ and 0.38 in the upper and lower graphs, respectively (after Ando and Yamashita, 2007)

with the main fault without arresting. This suggests that complicated fault geometry can be generated dynamically. It is also shown in the figure that the emergence of such a large-scale branch tends to suppress the growth of the next nucleated branches in its neighborhood, which is due to the stress shadow effect as will be discussed below. Such phenomena are also observed in Figure 3, which shows an example of longer simulation time.

Figure 4 shows the extending velocity of the tip of main fault soon attains a constant value with the extension. Comparing the velocity at each position between this multiple branch model and the case of a planar fault, we can clearly see that the dynamic branching reduces the rupture velocity of the main fault; the assumed physical parameters are identical between them (except the geometry). This data will contribute to the formation of approximately self-similar shapes for the spatial distribution of meso-branches.

We further investigate the effect of mesoscopic branches as the stress-slip relationship on a macroscopic scale, neglecting the thickness of the fault zone. Figure 5 shows this relationship obtained at each point along the main fault by calculating shear stress and displacement immediately outside the fault zone. The critical slip weakening distance $D_{c}$ and the fracture energy $G_{c}$ become proportional to the

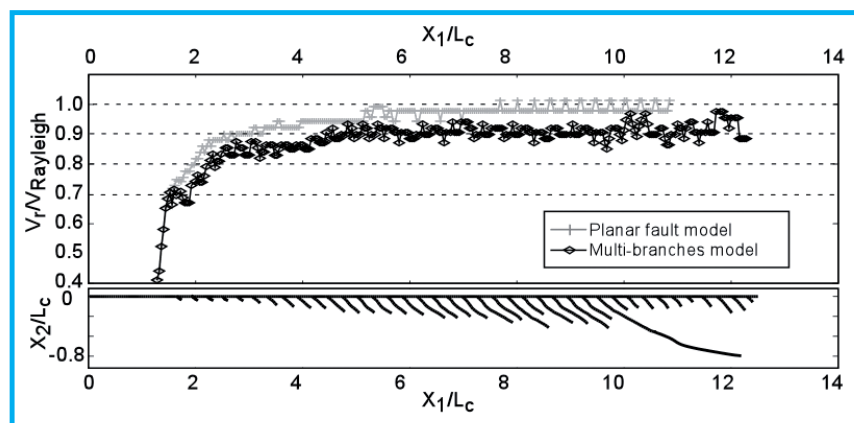

Figure 4. Reduction of extending velocity of the tip of main fault to approximately a constant sub-Rayleigh value due to multiple branching; the model shown in Figure 4 is assumed. (Upper) Black curve denotes the fault tip velocity in the multi-branch model. Gray curve shows the case of planar fault model with no branches; the other assumptions are the same as in the multibranch model. (Lower) Thick curves denote the corresponding fault geometry of the multi-branch model (after Ando and Yamashita, 2007).

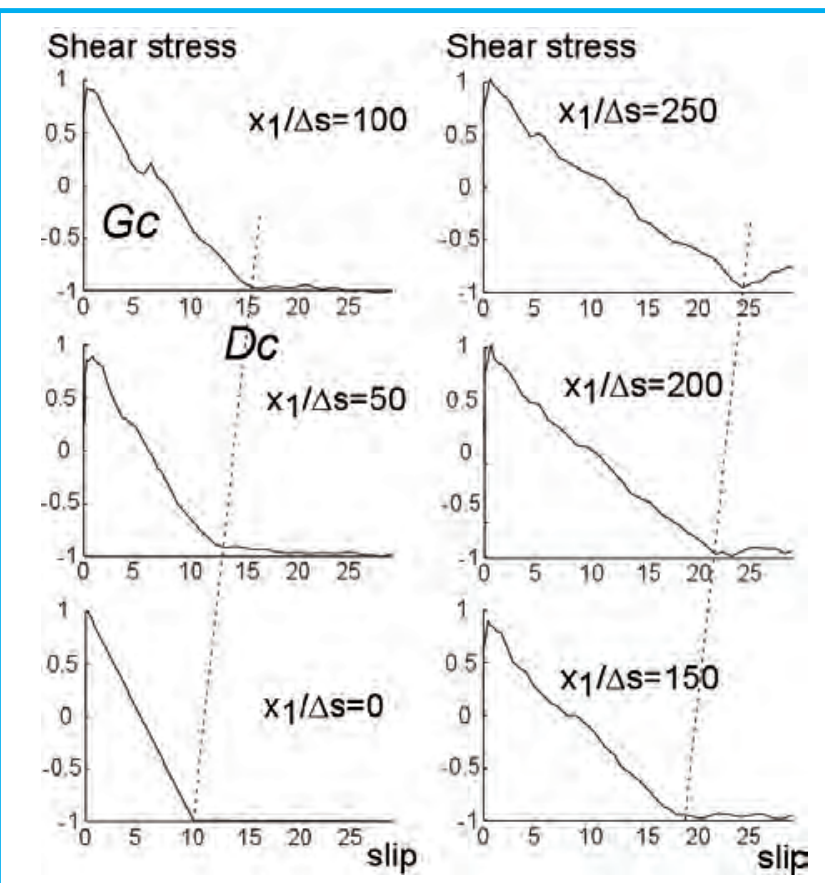

Figure 5. Slip-shear stress relationship in macroscopic scale. Each pane shows the shear stress as the function of slip obtained for equally Ispaces sampling points along main fault. The dimension of the triangle corresponds the fracture energy $\mathrm{G}_{\mathrm{c}}$ (after Ando, 2005).

distance of rupture propagation. This explains why the observed constant rupture velocity (Fig.4) decreased under a sub-Rayleigh speed. Note that this simple scaling is satisfied as long as the rupture propagation distance is smaller than a critical length $L_{m}$.

\section{References}

Ando, R. and Yamashita, T., 2007. Effects of mesoscopic-scale fault structure on dynamic earthquake ruptures: Dynamic formation of geometrical complexity of earthquake faults, J. Geophys. Res., 112, B09303, doi:10.1029/ $20062006 J$ JB004612.

Ando, R., 2005. Development of efficient spatio-temporal boundary integral equation method and theoretical study on dynamics of fault zone formation and earthquake ruptures (in Japanese), Univ. of Tokyo, Tokyo.

\section{Authors}

Ryosuke Ando, Natonal Research Institute for Earth Science and Disaster Prevention, 3-1 Tennodai, Tsukuba, Ibaraki, 305-0006, Japan, e-mail:ando@bosai.go.jp.

Teruo Yamashita, Earthquake Research Institute, The University of Tokyo, 1-1-1 Yayoi, Bunkyo-ku, Tokyo, Japan. 